\title{
DISAPPEARANCE OF SYMPTOMATIC VENOUS THROMBOSIS AFTER NEONATAL CARDIAC OPERATIONS DURING ANTITHROMBIN III SUBSTITUTION
}

\author{
Jari Petäjä, MD, PhD, Kaija Peltola, MD, PhD, and Paula Rautiainen, MD, PhD, Helsinki, Finland
}

Venous thrombosis (VT) of the central intrathoracic veins is a significant complication after cardiac operations. ${ }^{1}$ In neonates, secondary antithrombin III (AT III) deficiency is one component of a complicated postoperative coagulopathy contributing to the thrombogenesis. ${ }^{2-4}$ We previously suggested that substitution therapy with AT III concentrate might be a viable approach to reducing the incidence of $\mathrm{VT}^{2}$ Accordingly, we now report our experience after 3 years of applying a systematic AT III substitution policy in postoperative neonatal care. It seems that AT III substitution helps to avoid symptomatic VT.

Patients and methods

AT III substitution policy. Since March 1995, systematic daily follow-up of plasma AT III levels was recommended in the Children's Hospital, University of Helsinki, during the postoperative intensive care of neonates who have undergone cardiac operations. Likewise, substitution with AT III concentrate (Atenative, Pharmacia \& Upjohn, Finland) for levels below $50 \%$ of the adult mean was encouraged. The dose was calculated on the basis of the AT III level but was typically about $50 \mathrm{IU} / \mathrm{kg}$, with the aim of a postinfusion AT III level between $80 \%$ and $100 \%$. However, the decision to both measure AT III and prescribe the substitute was left to the anesthesiologist responsible for the particular patient. The result was $90 \%$ coverage of eligible patients (see below) and minor variation in the AT III levels used as the indication for AT III substitution.

Historical controls. The control group consisted of 160 neonates who underwent cardiac operations between January 1985 and February 1995.

Study cohort. Between March 1995 and June 1998, 102 neonates underwent a cardiac operation. During this period, an active operative approach was also applied to patients with severe hypoplastic left heart syndrome. Eleven such patients died during the immediate perioperative period before first postoperative AT III measurement, and these patients were excluded from the study cohort. An additional group of 9 surviving patients was excluded from the study because AT III was not measured during the postoperative intensive care period. The study cohort thus consisted of 82 neonates.

From Children's Hospital, University of Helsinki, Helsinki, Finland. Received for publication June 17, 1999; accepted for publication July 7, 1999.

Address for reprints: Jari Petäjä, MD, PhD, Children's Hospital, University of Helsinki, Stenbäckinkatu 11, FIN-00290 Helsinki, Finland.

J Thorac Cardiovasc Surg 1999;118:955-6

Copyright $(\odot) 1999$ by Mosby, Inc.

$0022-5223 / 99 \$ 8.00+0 \quad \mathbf{1 2 / 5 4 / 1 0 1 2 8 5}$

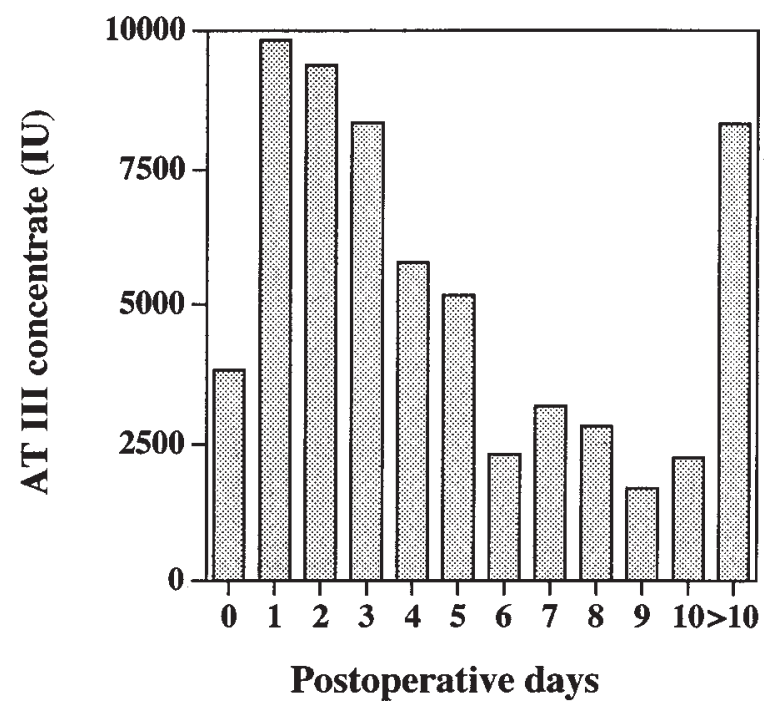

Fig 1. The administration of antithrombin III (AT III) concentrate in the cohort of 82 neonates undergoing cardiac operations. On the basis of plasma AT III determinations, 14 patients did not receive AT III concentrate. Sixty-one percent of the cumulative dose was given between postoperative days 1 and 5. Day 0 indicates the day of operation.

Phlebography was performed if symptoms of venous stasis were suspected. The right atrium was repeatedly visualized in routine postoperative cardiac echocardiograms.

Results. The plasma AT III level was measured 455 times in the study cohort. The AT III level was below $41 \%$ of the adult mean (lower limit of normal for neonates) 5 in $47(57 \%)$, $14(17 \%)$, and $14(17 \%)$ during postoperative days 1 to 3,4 to 6 , and 7 to 10 , respectively. Using the $50 \%$ cutoff for secondary AT III deficiency, the frequencies were 68 (83\%), 33 (40\%), and 24 (29\%) during postoperative days 1 to 3,4 to 6 , and 7 to 10 , respectively. Sixty-eight $(83 \%)$ patients received a total of 62,965 IU of AT III concentrate (mean \pm standard deviation $768 \pm 119$ IU per cohort patient) (Fig 1).

In the historical controls, 10 of the 160 neonates had central VT as described in detail earlier ${ }^{1}$ (Table I). In all 10 patients, VT was considered clinically significant according to the following criteria: (1) VT caused $(n=1)$ or significantly contributed $(n=$ 3) to the death of the patient. (2) The patient survived, but VT caused symptoms of superior vena cava syndrome $(n=3)$. (3) On the basis of the judgment of the physician responsible, the extent and location of VT necessitated thrombolytic treatment $(\mathrm{n}=3)$. The thrombi were diagnosed during the 10 years as follows: $1988, \mathrm{n}=2 ; 1990, \mathrm{n}=1 ; 1992, \mathrm{n}=1 ; 1993, \mathrm{n}=3 ; 1994, \mathrm{n}$ 
Table I. Antithrombin III (AT III) substitution in neonates undergoing cardiac operations

\begin{tabular}{lcll}
\hline & \multicolumn{3}{c}{ AT III } \\
& \multicolumn{1}{c}{ Before } & substitution & P value \\
\hline VT & $10 / 160(6.3 \%)$ & $2^{*} / 82(2.3 \%)$ & .20 \\
Clinically significant $\mathrm{VT}^{\dagger}$ & $10 / 160(6.3 \%)$ & $0 / 82(0 \%)$ & .021 \\
VT-associated mortality & $4 / 160(2.5 \%)$ & $0 / 82(0 \%)$ & .15 \\
\hline
\end{tabular}

Before indicates operations carried out before March 1995, when AT III substitution was not used, and AT III substitution indicates the operations during the AT III substitution policy as explained in the "Patients and methods."

*Both patients with asymptomatic VT had plasma AT III levels below $51 \%$ of the adult mean on several days preceding the detection of VT despite AT III substitution.

'For definition of "Clinically significant VT" see text. The $P$ value was calculated by the $\chi^{2}$ test (1-tailed).

$=2 ; 1995, \mathrm{n}=1$. The overall postoperative mortality was 22 deaths $(15 \%)$ among 150 patients without VT and 4 deaths (40\%) among 10 patients with VT.

In the study cohort, no symptomatic VT was observed (Table I). Within this cohort the overall postoperative mortality was 14 deaths (17\%) among 82 patients.

In a patient with transposition of the great arteries (TGA) and the arterial switch operation, a small mural thrombus in the right atrium was observed by echocardiography on postoperative day 14 . No treatment was considered necessary, and the patient made an uneventful recovery. Despite AT III substitution, however, the patient had an AT III level below 51\% on 7 occasions between postoperative days 1 and 9. In another patient undergoing arterial switch for TGA, the postoperative course was first complicated by cardiac tamponade after removal of a catheter from the left pulmonary artery on postoperative day 3. After the resternotomy, the patient had signs of a septic infection. On postoperative day 9, his condition deteriorated and an abdominal catastrophe was suspected. Strangulation of the small intestine was discovered at laparotomy. Extensive intestinal resection was performed, but immediately after the operation his hemodynamic condition deteriorated and the patient died. An autopsy showed severe damage to the remaining gut, and changes consistent with ischemic multiorgan failure were also evident in the kidneys and liver. The abdominal arterial and venous circulations were free of thrombi. A fresh, short VT was discovered in the superior vena cava. It was concluded that the patient died of the intestinal catastrophe unrelated to the VT. Despite AT III substitution, plasma AT III was below $51 \%$ on 5 occasions between postoperative days 1 and 7 . The lowest AT III level in this patient was $18 \%$ on postoperative day 4 , even though he received an $83 \mathrm{IU} / \mathrm{kg}$ dose of AT III concentrate on day 3.

Discussion. AT III substitution for secondary postoperative AT III deficiency in neonatal cardiac operations was associated with abrupt disappearance of symptomatic VT. Our reasons for undertaking this practice were multiple. First, previ- ous work showed that neonates had up to a 10-fold risk of VT compared with older children undergoing cardiac operations. ${ }^{1}$ Second, thrombogenesis in this patient group was associated with multiple prothrombotic alterations in coagulation, including a transient AT III deficiency preceding symptomatic VT. ${ }^{2}$ Third, little could be done to reduce the thrombogenic burden caused by indwelling catheters, surgical trauma, and postoperative hemodynamic changes. Research proof of both efficacy and safety of alternative VT prophylaxis by heparin or oral anticoagulants in neonates is also lacking. Finally, AT III substitution was feasible because of the transient nature of postoperative AT III decrease and the availability of a virus-inactivated concentrate.

In the absence of observed AT III-induced bleeding complications, cost-benefit becomes a central issue. At Finnish prices for AT III concentrate and AT III determinations, the estimated cost of the AT III substitution for the study cohort was $\$ 40,000$ (US currency). The figures in Table I show that this should be compared with the estimated benefit of avoiding 5 clinically significant VTs. Since $\$ 8000$ (US currency) per avoided VT corresponds to the cost of 4 to 5 days of intensive care, we believe that AT III substitution for the cohort of 82 neonates was medically beneficial in a cost-neutral way.

Since the current study was neither randomized nor blinded, several possible biases exist, but for certain central issues bias is unlikely. First, since all neonatal cardiac operations in Finland are performed at the Children's Hospital, there is no patient selection bias. If anything, more moribund patients like those with severe hypoplastic left heart syndrome have recently been included. Second, no major changes in the perfusion techniques or the perfusion team have taken place since 1995. Finally, no significant changes concerning the types and care (including heparinization of the infused solutions) of central venous catheters have taken place during the 1990s. Thus we hope that our observational data and experience prove sufficiently provocative to stimulate randomized VT prophylaxis studies in neonatal cardiac surgery.

\section{REFERENCES}

1. Petäjä J, Lundström U, Sairanen H, Marttinen E, Griffin JH. Central venous thrombosis after cardiac surgery in children. J Thorac Cardiovasc Surg 1996;112:883-9.

2. Petäjä J, Peltola K, Sairanen H, Leijala M, Kekomäki R, Vahtera E, et al. Fibrinolysis, antithrombin III, and protein $\mathrm{C}$ in neonates during cardiac operations. J Thorac Cardiovasc Surg 1996;112:665-71.

3. Kern FH, Morana NJ, Sears JJ, Hickey PR. Coagulation defects in neonates during cardiopulmonary bypass. Ann Thorac Surg 1992;54:541-6.

4. Boldt J, Knothe C, Schindler E, Welters A, Dapper F, Hempelmann G. Thrombomodulin in pediatric cardiac surgery. Ann Thorac Surg 1994;57:1584-9.

5. Andrew M, Paes B, Johnston M. Development of the hemostatic system in the neonate and young infant. Am J Pediatr Hematol Oncol 1990;12:95-104. 